\title{
NEW PARAMETRIC 3D SNAKE FOR MEDICAL SEGMENTATION OF STRUCTURES WITH CYLINDRICAL TOPOLOGY
}

\author{
Daniel Schmitter ${ }^{1}$, Christophe Gaudet-Blavignac ${ }^{1}$, Davide Piccini ${ }^{2,3}$, and Michael Unser ${ }^{1}$ \\ ${ }^{1}$ Biomedical Imaging Group, École polytechnique fédérale de Lausanne (EPFL), Switzerland \\ ${ }^{2}$ Advanced Clinical Imaging Technology Group, Siemens Healthcare Sector, Renens, Switzerland \\ ${ }^{3}$ Center for Biomedical Imaging (CIBM), Lausanne, Switzerland
}

\begin{abstract}
We propose a new parametric 3D snake with cylindrical topology. Its construction is based on interpolatory basis functions which facilitates user-interaction because the control points of the snake directly lie on the surface of the deformable cylinder. We prove that the basis functions exactly reproduce a cylinder and propose a new parametrization as a tensorproduct spline surface. We provide explicit formulas for the energy function based on Green's theorem that speed up the computation of the optimization algorithm. We have implemented the proposed framework as a freely available opensource plugin for the bioimaging platform Icy. Its utility has been tested on phantom data as well as on real 3D data to segment the spinal cord and the descending aorta.
\end{abstract}

Index Terms - spline snakes, splines, active contours, 3D segmentation, medical, aorta

\section{INTRODUCTION}

The development of methods for the assessment of physiological structures and measurements using 3D imaging modalities is an active field of research. They are crucial for correct diagnosis of diseases, risks or malformations. The segmentation of complex structures in 3D is difficult without making use of a template [1]. In that case one needs to deal with the problem of how to construct an accurate atlas representing a whole patient population. For anatomical structures where the shape is simpler, such as the aorta, spinal cord, vena cava or the coronary arteries, which are topologically equivalent to a cylinder, this prior information can be exploited to design specialized segmentation algorithms. This motivates the development of a deformable model with few degrees of freedom. Existing methods for the segmentation of vascular structures often rely on a large number of parameters, such as mesh- [2], tensor- [4] or tracking [4]- and path minimization [5]-based models.

This work was funded the Swiss National Science Foundation under Grant 200020-144355, the Center for Biomedical Imaging of the GenevaLausanne Universities and EPFL, as well as by the Foundations Leenaards and Louis-Jeannet.
We propose a new method for the segmentation of structures with cylinder-like topology such as the aorta or the spinal cord. Our method is atlas-free and accounts for the possibility of user-interaction. It relies on a new parameterization of the cylinder using compactly supported basis functions and the resulting shape is smooth by construction. We show that the bases perfectly reproduce the cylinder as a tensor-product spline surface. Furthermore, they are interpolatory which implies that the control points of the shape, which are accessible to the user, directly lie on the surface; a property that allows intuitive and easy user-interaction because each basis function is associated with a control point on the surface. Our model has the advantage that much fewer parameters need to be used for the representation of the shape as opposed to mesh-based methods $[6,7,8]$. This allows a fast and robust optimization because it constraints the space of the possible segmentation solutions. Using the new proposed parametric surface, we construct a 3D snake and provide an explicit expression for a contour-based energy function that attracts the snake towards the boundary of interest $[9,10,11]$. The provided energy function enables an explicit computation of its gradient; a property that can be exploited for an efficient implementation. Finally, we have tested the robustness w.r.t. to noise on test data and we have performed an evaluation on real MRI data using a cohort of 14 healthy subjects [12] to segment the descending thoracic aorta; a region that is of interest to measure hemodynamics after thoracic endovascular aortic repair as well as aneurisms [13] (see Figure 1). Our method applies to 3D MRI as well as to CT images.

\section{NEW PARAMETRIZATION OF THE CYLINDER}

In this section we present a new parametrization of the cylinder as a tensor-product spline surface. For this purpose our basis functions need to be able to reproduce circles and ellipses as well as first degree polynomials.

\subsection{Reproduction of circles and ellipses}

We consider the following basis function: 

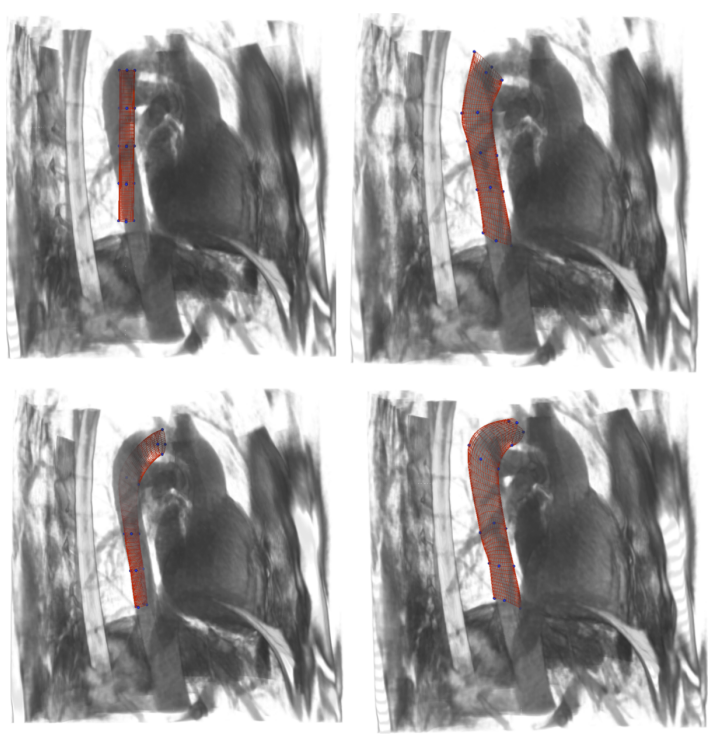

Fig. 1. Segmentation of the aorta with the cylinder snake in 3D MRI. The red wireframe represents the surface of the snake and the blue dots are the control points.

$$
\phi(t)=\lambda_{1} \beta_{\boldsymbol{\alpha}_{1}}(t)-\lambda_{2}\left[\beta_{\boldsymbol{\alpha}_{2}}(t)+\beta_{\boldsymbol{\alpha}_{2}}(t-1)\right],
$$

where

$$
\begin{gathered}
\lambda_{1}(M)=\frac{2 \pi^{3}}{M^{2}\left(M \sin \left(\frac{2 \pi}{M}\right)-\pi \cos \left(\frac{2 \pi}{M}\right)-\pi\right)} \\
\lambda_{2}(M)=\frac{\pi^{2}\left(2 \pi-M \sin \left(\frac{2 \pi}{M}\right)\right)}{M^{2}\left(\cos \left(\frac{2 \pi}{M}\right)-1\right)\left(M \sin \left(\frac{2 \pi}{M}\right)-\pi \cos \left(\frac{2 \pi}{M}\right)-\pi\right)}
\end{gathered}
$$

and $\hat{\beta}_{\boldsymbol{\alpha}}(\omega)=\prod_{k=1}^{n} \frac{1-e^{\alpha_{k}-j \omega}}{j \omega-\alpha_{k}}$ is the $n$-th order causal exponential B-spline defined in the Fourier domain and the poles are given by $\boldsymbol{\alpha}_{1}=\left(0,0, \frac{j 2 \pi}{M},-\frac{j 2 \pi}{M}\right)$ and $\boldsymbol{\alpha}_{2}=\left(0, \frac{j 2 \pi}{M},-\frac{j 2 \pi}{M}\right)$.

Proposition 1. The basis $\varphi_{1}=\phi(\cdot+2)$ is an interpolator and is capable of reproducing the complex exponentials $e^{j 2 \pi t}$ and $e^{-j 2 \pi t}$ independent of the number of control points $M$.

Proof: In order to show that $\varphi_{1}$ is an interpolator we notice that the $n$-th order exponential B-spline has support $n$. Thus, $\varphi_{1}$ has a support equal to 4 . By imposing the corrsponding interpolation conditions on (1) and solving for $\lambda_{1}$ and $\lambda_{2}$ we obtain the weights given by (2) and (3). In order to prove the reproduction properties of $\varphi_{1}$ we use the exponential reproduction properties from the exponential B-spline, i.e. if $\alpha \in \boldsymbol{\alpha}$, then there exists a sequence $p[k]$ such that $e^{\alpha t}=\sum_{k \in \mathbb{Z}} p[k] \beta_{\boldsymbol{\alpha}}(t-k)$ [14]. Therefore, considering $\alpha \in \boldsymbol{\alpha}_{2} \subset \boldsymbol{\alpha}_{1}$ we obtain

$$
\begin{aligned}
& \sum_{k \in \mathbb{Z}} p[k]\left[\lambda_{1} \beta_{\boldsymbol{\alpha}_{2}}(t-k)-\lambda_{2}\left(\beta_{\boldsymbol{\alpha}_{2}}(t-k)+\beta_{\boldsymbol{\alpha}_{2}}(t-k-1)\right)\right] \\
& =\lambda_{1} e^{\alpha t}-\lambda_{2}\left(e^{\alpha t}+e^{\alpha(t-1)}\right) \\
& =e^{\alpha t}\left(\lambda_{1}-\lambda_{2}\left(1+e^{-\alpha}\right)\right)
\end{aligned}
$$

From [14] (Proposition 2) we know that if a function $\psi$ reproduces exponential polynomials then $\tilde{\psi} * \psi$ also reproduces these exponential polynomials if $\tilde{\psi}$ satisfies some mild conditions. Thus, since $\beta_{\boldsymbol{\alpha}_{1}}=\beta_{0} * \beta_{\boldsymbol{\alpha}_{2}}$ and

$$
\begin{aligned}
& \sum_{k \in \mathbb{Z}} \phi(t-k)= \\
& \sum_{k \in \mathbb{Z}} p[k]\left[\lambda_{1}\left(\beta_{0} * \beta_{\boldsymbol{\alpha}_{2}}\right)(t-k)-\lambda_{2}\left(\beta_{\boldsymbol{\alpha}_{2}}(t-k)+\beta_{\boldsymbol{\alpha}_{2}}(t-k-1)\right)\right]
\end{aligned}
$$

we see that $\phi$ also reproduces the exponential polynomials given by (4). By replacing $t$ by $t+2$ in (4) we directly see that $\varphi_{1}$ has the same reproduction properties as $\phi$. By choosing $\alpha= \pm \frac{j 2 \pi}{M}$ we directly obtain the complex exponentials described by Proposition 1.

Corollary 1. The interpolator $\varphi_{1}$ reproduces $\cos (2 \pi t)$ and $\sin (2 \pi t)$ independent of the number of control points $M$.

Proof: By exploiting the exponential reproduction property of B-splines and the property of $\varphi_{1}$ of being an interpolator we write

$\cos \left(\frac{2 \pi t}{M}\right)=\frac{e^{\frac{j 2 \pi t}{M}}+e^{-\frac{j 2 \pi t}{M}}}{2}=\sum_{k \in \mathbb{Z}}\left[\frac{e^{\frac{j 2 \pi k}{M}}+e^{-\frac{j 2 \pi k}{M}}}{2}\right] \varphi_{1}(t-k)$

and therefore

$$
\cos (2 \pi t)=\sum_{k \in \mathbb{Z}} \cos [2 \pi k] \varphi_{1}(M t-k)
$$

In a similar way we obtain $\sin (2 \pi t)$.

Plots of the reconstructed trigonometric functions are shown in Figure 2 as well as the circle $\boldsymbol{r}$ obtained through the parametric equation $\boldsymbol{r}(t)=(\cos (2 \pi t), \sin (2 \pi t))$.

\subsection{Reproduction of $\mathbf{1}^{\text {st }}$ degree polynomials}

The parametric representation of the cylinder as a spline surface requires that at least one basis function reproduces $1^{\text {st }}$ degree polynomials. We use the Keys interpolator [15] which is in $\mathcal{C}^{1}$ and reproduces $2^{\text {nd }}$ degree polynomials. It is given by

$$
\varphi_{2}(t)=\left\{\begin{array}{ll}
\frac{1}{2}\left(2-t^{2}(3 t+5)\right) & -1<t \leqslant 0 \\
-\frac{1}{2}(t-2)^{2}(t-1) & 1 \leqslant t<2 \\
\frac{1}{2}(t+1)(t+2)^{2} & -2<t \leqslant-1 \\
\frac{1}{2}\left((3 t-5) t^{2}+2\right) & 0<t<1
\end{array} .\right.
$$



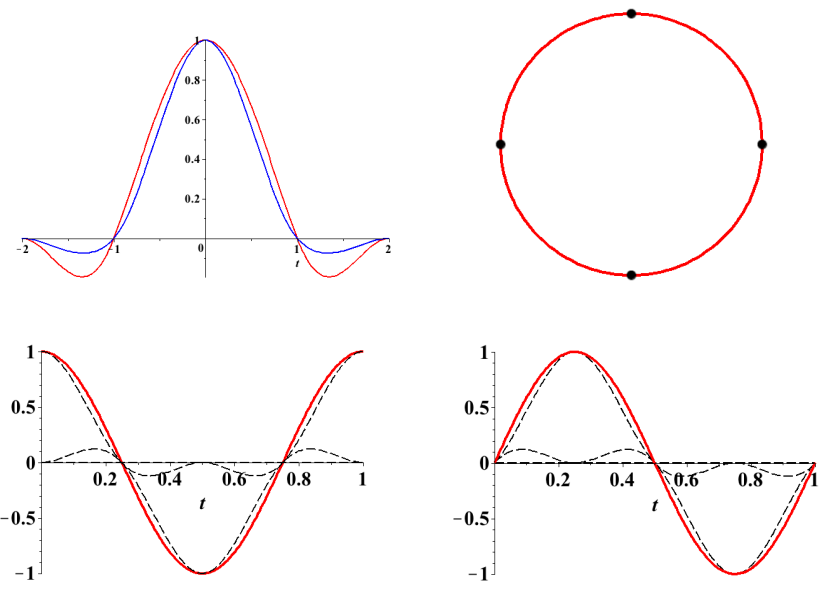

Fig. 2. Top left: Keys interpolator (blue) and the proposed ellipse reproducing interpolator (red). Top right: the circle obtained with the parametric equation $\boldsymbol{r}(t)=$ $(\cos (2 \pi t), \sin (2 \pi t))$. Bottom: $\cos (2 \pi t)$ (left) and $\sin (2 \pi t)$ (right) are shown together with the basis functions for $M=3$.

Its support is also equal to 4 as for $\varphi_{1}$. A plot of the line that has been reproduced with $\varphi_{2}$ is shown in Figure 3 .

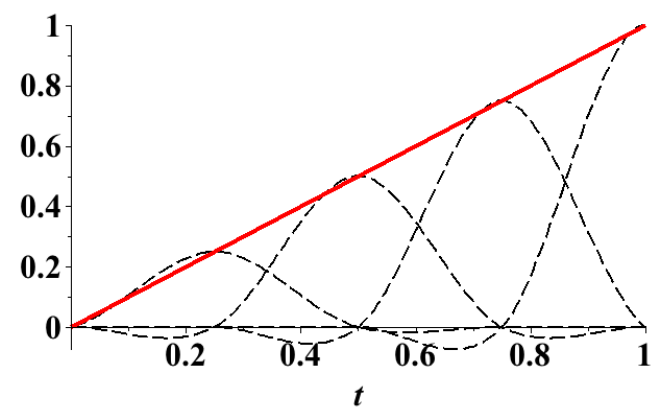

Fig. 3. Reproduction of the line and weighted basis functions.

\subsection{Reproduction of the cylinder}

Proposition 2. The normalized cylinder surface can be expressed as

$$
\boldsymbol{\sigma}(u, v)=\sum_{k=0}^{M-1} \sum_{k=-1}^{M+1} \mathbf{c}[k, l] \varphi_{1, M}(M u-k) \varphi_{2}(M v-l),
$$

where $u, v \in[0,1]$ and $\varphi_{1, M}$ is the M-periodization of $\varphi_{1}$ and the control points of the surface are given by

$$
c[k, l]=\left(\begin{array}{c}
\cos \left[\frac{2 \pi k}{M}\right] \\
\sin \left[\frac{2 \pi k}{M}\right] \\
\frac{l}{M}
\end{array}\right) .
$$

Proof: By using the $M$-periodized basis function of $\varphi_{1}$

$$
\varphi_{1, M}(M t-k)=\sum_{n=-\infty}^{+\infty} \varphi_{1}(M(t-n)-k) \text {, we can re- }
$$

express the cosine in (5) as

$$
\cos (2 \pi t)=\sum_{k=0}^{M-1} \cos \left[\frac{2 \pi k}{M}\right] \varphi_{1, M}(M t-k) . \text { In a similar }
$$

way we obtain

$$
\sin (2 \pi t)=\sum_{k=0}^{M-1} \sin \left[\frac{2 \pi k}{M}\right] \varphi_{1, M}(M t-k) . \text { The reproduc- }
$$

tion of the straight line can be expressed as $\sum_{k \in \mathbb{Z}} \frac{k}{M} \varphi_{2}(M t-$ $k$ ). If we enforce $t$ to lie in the interval $[0,1]$ and because the support of $\varphi_{2}$ is limited to $[-2,2]$ the summation can be restricted to the indices $k \in[-1, M+1]$. Since $\varphi_{1}$ and $\varphi_{2}$ satisfy the partition of unity condition, i.e. $\sum_{k=-\infty}^{\infty} \varphi(t-k)=1$ (proof ommitted) we can develop the standard parametrization of the cylinder for $u, v \in[0,1]$ as follows:

$$
\begin{aligned}
\boldsymbol{\sigma}(u, v) & =\left(\begin{array}{c}
\cos (2 \pi u) \\
\sin (2 \pi u) \\
v
\end{array}\right) \\
& =\sum_{k=0}^{M-1}\left(\begin{array}{c}
\cos \left[\frac{2 \pi k}{M}\right] \\
\sin \left[\frac{2 \pi k}{M}\right] \\
1
\end{array}\right) \varphi_{1, M}(M u-k) \cdot \sum_{k=-1}^{M+1}\left(\begin{array}{c}
1 \\
1 \\
\frac{k}{M}
\end{array}\right) \varphi_{2}(M v-k) \\
& =\sum_{k=0}^{M-1} \sum_{k=-1}^{M+1}\left(\begin{array}{c}
\cos \left[\frac{2 \pi k}{M}\right] \\
\sin \left[\frac{2 \pi k}{M}\right] \\
\frac{k}{M}
\end{array}\right) \varphi_{1, M}(M u-k) \varphi_{2}(M v-l)
\end{aligned}
$$

An important property of the surface that we use to construct the snake is that it must be able to outline shapes irrespective of their size, orientation and position. It must therefore be invariant to affine transformations, i.e.

$$
\begin{aligned}
& \mathbf{A} \boldsymbol{\sigma}(u, v)+\mathbf{b} \\
& =\sum_{k=0}^{M-1} \sum_{l=-1}^{M+1}(\mathbf{A} \boldsymbol{c}[k, l]+\mathbf{b}) \varphi_{1, M}(M u-k) \varphi_{2}(M v-l),
\end{aligned}
$$

where $\mathbf{A}$ is a $3 \times 3$ matrix and $\mathbf{b} \in \mathbb{R}^{3}$. It is easy to see that equation (7) is automatically satisfied if the basis functions $\varphi_{1}$ and $\varphi_{2}$ satisfy the partition of unity. A plot of the resulting surface is shown in Figure 4 (left).

\section{3D PARAMETRIC SPLINE SNAKE}

To construct the snake we need to define an energy functional that can be minimized in order to attract the snake surface towards the boundary of interest. We use a gradient-based energy similar to the one proposed by [9]. It is given by 

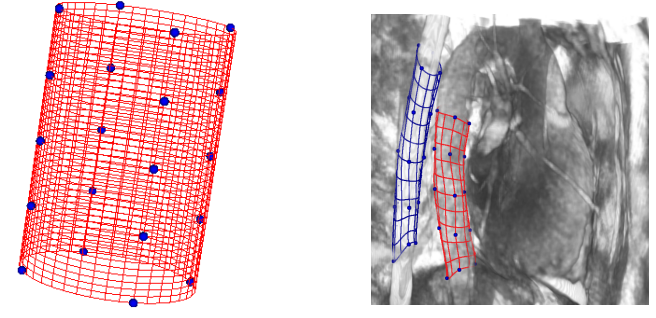

Fig. 4. Cylinder snake. Left: wireframe representation of the cylinder created as a tensor-product spline surface given by Proposition 2. The blue points are the control points. Right: simultaneous segmentation of the spinal cord (blue) and the thoracic descending aorta (red).

$$
\begin{aligned}
E_{\text {grad }} & =-\iint_{\partial \Omega} \Delta^{x} \mathrm{~d} y \wedge \mathrm{d} z \\
& =-\int_{0}^{1} \int_{0}^{1} \Delta^{x}(\boldsymbol{\sigma}(u, v))\left(\frac{\partial y}{\partial u} \frac{\partial z}{\partial v}-\frac{\partial y}{\partial v} \frac{\partial z}{\partial u}\right) \mathrm{d} u \mathrm{~d} v
\end{aligned}
$$

where $\Delta^{x}(x, y, z)=\int_{-\infty}^{x} \Delta(\tau, y, z) \mathrm{d} \tau$ can be preintegrated and stored in a lookup table in order to speed up the computational process. The partial derivatives in (8) are computed through

$$
\frac{\partial \boldsymbol{\sigma}}{\partial u}(u, v)=M \sum_{k=0}^{M-1} \sum_{l=-1}^{M+1} \mathbf{c}[k, l] \dot{\varphi}_{1, M}(M u-k) \varphi_{2}(M v-l)
$$

$\frac{\partial \boldsymbol{\sigma}}{\partial u}(u, v)=M \sum_{k=0}^{M-1} \sum_{l=-1}^{M+1} \mathbf{c}[k, l] \varphi_{1, M}(M u-k) \dot{\varphi}_{2}(M v-l)$,

where $\frac{\partial \boldsymbol{\sigma}}{\partial u}=\left(\frac{\partial x}{\partial u}, \frac{\partial y}{\partial u}, \frac{\partial z}{\partial u}\right)$ and $\frac{\partial \boldsymbol{\sigma}}{\partial v}=\left(\frac{\partial x}{\partial v}, \frac{\partial y}{\partial v}, \frac{\partial z}{\partial v}\right)$.

Equation (8) allows to compute a closed-form expression of its gradient w.r.t. to the control points, which enables an efficient implementation of the optimization algorithm.

\section{EXPERIMENTS}

We have implemented and validated our proposed framework on artificial as well as on real 3D MRI data. For the phantom data, we created a perfect 3D hollow cylinder and corrupted the image with increasing levels of additive Gaussian white noise. The overlap between the initial position of the snake and the perfect cylinder corresponds to a Jaccard index of 0.12. The signal-to-noise ratios (SNR) and resulting overlap measures are shown in Table 1.
Table 1. Jaccard indices for segmentation of (noisy) data.

\begin{tabular}{cc}
\hline \hline SNR [dB] (stdd) & Jaccard index \\
\hline$\infty(-)$ & 0.94 \\
$9.91(10)$ & 0.94 \\
$0.27(30)$ & 0.94 \\
$-4.17(50)$ & 0.94 \\
$-7.10(70)$ & 0.92 \\
$-9.21(90)$ & 0.92 \\
\hline \hline
\end{tabular}

We have validated the snake on real data. A gold standard was created by manually segmenting by an expert clinician the thoracic descending aorta on 14 scans taken from healthy subjects. The mean overlap measures w.r.t. to the initial position and segmentation results are shown in Table 2 as well as the standard deviations (std).

Table 2. Mean Jaccard indices for segmentation of real data.

\begin{tabular}{c|c}
\hline \hline- & mean Jaccard index (std) \\
\hline initialization & $0.23(0.23)$ \\
result & $0.96(0.02)$ \\
\hline \hline
\end{tabular}

The optimization is carried out by a Powell-like line-search method [16]. The segmentation took less than 4 seconds on average on a $2.3 \mathrm{GHZ}$ processor with 8 GB RAM. Furthermore, we have also successfully tested the framework for computed tomography data and for the segmentation of the spinal cord in 3D MRI (results will be published elsewhere). An illustration of a real 3D MRI scan where the spinal cord and the thoracic descending aorta are segmented are shown in Figure 4 (right).

\section{CONCLUSIONS}

We have proposed a novel parametrization of the cylinder in order to construct a 3D snake. We have shown how to perfectly reproduce the cylindrical topology using interpolatory basis functions. This allows the construction of a tensorproduct spline surface where the control points lie on the surface itself; an advantage for user-interactive applications. We provided an explicit formulation for a gradient energy. The results obtained on real data are promising. Furthermore, our experiments show that the proposed algorithm is robust to noise. An implementation of the proposed framework is freely available as an open source plugin for Icy [17] at http://bigwww.epfl.ch/algorithms.html.

\section{REFERENCES}

[1] Cemil Kirbas and Francis Quek, "A review of vessel extraction techniques and algorithms," ACM Comput. 
Surv., vol. 36, no. 2, pp. 81-121, June 2004.

[2] B. De Leener, S. Kadoury, and J. Cohen-Adad, "Robust, accurate and fast automatic segmentation of the spinal cord," NeuroImage, vol. 98, no. 0, pp. 528 - 536, 2014.

[3] S. Cetin, A. Demir, A. Yezzi, M. Degertekin, and G. Unal, "Vessel tractography using an intensity based tensor model with branch detection," Medical Imaging, IEEE Transactions on, vol. 32, no. 2, pp. 348-363, Feb 2013.

[4] J. Lee, P. Beighley, E. Ritman, and N. Smith, "Automatic segmentation of $3 \mathrm{~d}$ micro-ct coronary vascular images," Medical Image Analysis, vol. 11, no. 6, pp. 630 - 647, 2007.

[5] H. Li and A. Yezzi, "Vessels as 4-d curves: Global minimal 4-d paths to extract 3-d tubular surfaces and centerlines," Medical Imaging, IEEE Transactions on, vol. 26, no. 9, pp. 1213-1223, Sept 2007.

[6] F. Precioso and M. Barlaud, "B-spline active contour with handling of topology changes for fast video segmentation.," EURASIP Journal on Applied Signal Processing, vol. 2002, no. 6, pp. 555-560, January 2002.

[7] D. Barbosa, T. Dietenbeck, J. Schaerer, J. D'hooge, D. Friboulet, and O. Bernard, "B-Spline explicit active surfaces: An efficient framework for real-time 3D region-based segmentation," IEEE Transactions on Image Processing, vol. 21, no. 1, pp. 241-251, January 2012.

[8] A. Dufour, R. Thibeaux, E. Labruyere, N. Guillen, and J.-C. Olivo-Marin, "3-D Active meshes: Fast discrete deformable models for cell tracking in 3-D time-lapse microscopy," IEEE Transactions on Image Processing, vol. 20, no. 7, pp. 1925-1937, July 2011.

[9] R. Delgado-Gonzalo, N. Chenouard, and M. Unser, "Spline-based deforming ellipsoids for interactive $3 \mathrm{~d}$ bioimage segmentation," IEEE Transactions on Image Processing, vol. 22, no. 10, pp. 3926-3940, October 2013.

[10] X. Bresson, S. Esedoglu, P. Vandergheynst, J.-P. Thiran, and S. Osher, "Fast global minimization of the active contour/snake model," Journal of Mathematical Imaging and Vision, vol. 28, no. 2, pp. 151-167, 2007.

[11] T.F. Chan and L.A. Vese, "Active contours without edges," IEEE Transactions on Image Processing, vol. 10, no. 2, pp. 266-277, February 2001.

[12] D. Piccini, P. Monney, C. Sierro, S. Coppo, G. Bonanno, R.B. van Heeswijk, J. Chaptinel, G. Vincenti, J. de Blois, Simon C. Koestner, T. Rutz, A. Littmann,
M.O. Zenge, J. Schwitter, and M. Stuber, "Respiratory self-navigated postcontrast whole-heart coronary mr angiography: Initial experience in patients," Radiology, vol. 270, no. 2, pp. 378-386, 2014.

[13] G.H.W. van Bogerijen, F. Auricchio, M. Conti, A. Lefieux, A. Reali, A. Veneziani, J.L. Tolenaar, F.L. Moll, V. Rampoldi, and S. Trimarchi, "Aortic hemodynamics after thoracic endovascular aortic repair, with particular attention to the bird-beak configuration," Journal of Endovascular Therapy, vol. 21, no. 6, pp. 791-802, January 2014.

[14] M. Unser and T. Blu, "Cardinal exponential splines: Part I-Theory and filtering algorithms," IEEE Transactions on Signal Processing, vol. 53, no. 4, pp. 14251438, April 2005.

[15] R. Keys, "Cubic convolution interpolation for digital image processing," Acoustics, Speech and Signal Processing, IEEE Transactions on, vol. 29, no. 6, pp. 11531160, Dec 1981.

[16] W.H. Press, S.A. Teukolsky, W.T. Vetterling, and B.P. Flannery, Numerical Recipes: The Art of Scientific Computing, Cambridge University Press, third edition, 1986.

[17] F. de Chaumont, S. Dallongeville, N. Chenouard, N. Hervé, S. Pop, T. Provoost, V. Meas-Yedid, P. Pankajakshan, T. Lecomte, Y. Le Montagner, T. Lagache, A. Dufour, and J.-C. Olivo-Marin, "Icy: An open bioimage informatics platform for extended reproducible research," Nature Methods, vol. 9, no. 7, pp. 690-696, July 2012. 